\title{
Estudo da PNPIC e da PNPMF e seus reflexos no Estado do Rio de Janeiro'
}

\section{Study of PNPIC and PNPMF and their reflections in Rio de Janeiro State}

\author{
${ }^{2}$ Fabiola Angelita Cezarina Martins, ${ }^{3}$ Glauco De Kruse Villas Bôas, ${ }^{4}$ Leandro Machado Rocha \\ ${ }^{1}$ Monografia apresentada junto ao Curso de Pós-Graduação Lato Sensu de Especialização em Gestão da Inovação em Fitomedicamentos, \\ do Instituto de Tecnologia de Fármacos, Farmanguinhos/FIOCRUZ. \\ ${ }^{2}$ Secretaria Municipal de Saúde de Volta Redonda (SMS-VR). \\ ${ }^{3}$ Núcleo de Gestão em Biodiversidade e Saúde (NGBS), Farmanguinhos/FIOCRUZ. \\ ${ }^{4}$ Universidade Federal Fluminense, Centro de Ciências Médicas, Laboratório de Tecnologia de Produtos Naturais \\ Correspondência: fabiola.acbm@gmail.com
}

\section{Resumo}

Em 2006, duas importantes políticas foram publicadas para a área de plantas medicinais e fitoterápicos: a Política Nacional de Práticas Integrativas e Complementares no SUS (PNPIC) e a Política Nacional de Plantas Medicinais e Fitoterápicos (PNPMF). O objetivo desse trabalho é apresentar, de forma sucinta e sistematizada, aspectos dessas duas políticas públicas, através de uma abordagem exploratória descritiva. Utilizou-se como fonte um levantamento dos documentos que definem as PNPIC e PNPMF.

Palavras-chave: políticas públicas; saúde; medicamentos fitoterápicos.

\begin{abstract}
In 2006, two important policies were published in the area of medicinal plants and herbal medicines: the Política Nacional de Práticas Integrativas e Complementares no SUS (PNPIC) and the Política Nacional de Plantas Medicinais e Fitoterápicos (PNPMF). The aim of this study is to present briefly systematization of the aspects of these two public policies through a descriptive exploratory approach. It was used as a survey of source documents defining the PNPIC and PNPMF.
\end{abstract}

Keywords: public policies; health; herbal medicines. 


\section{Introdução}

A magnitude da biodiversidade brasileira, conjunto de todos os seres vivos com sua variabilidade genética integral, não é conhecida com precisão, tal a sua complexidade, estimando-se mais de dois milhões de espécies distintas de plantas, animais e microorganismos. Isso coloca o Brasil como detentor da maior biodiversidade biológica do mundo (Wilson, 1997). Apesar disso e de toda a diversidade de espécies existentes, o potencial de uso de plantas como fonte de novos medicamentos é ainda pouco explorada. Em relação ao uso médico, estima-se que apenas 5 mil espécies foram estudadas (Rates, 2001).

A Organização Mundial de Saúde (OMS), considerando as plantas medicinais como importante instrumento da assistência farmacêutica, por meio de vários comunicados e resoluções, expressa sua posição a respeito da necessidade de valorizar a sua utilização no âmbito sanitário ao observar que $70 \%$ a 90\% da população de países em desenvolvimento dependem dessas plantas no que se refere à Atenção Primária à Saúde (OMS, 2011). Em alguns países industrializados, o uso de produtos da medicina tradicional é igualmente significante, como Canadá, França, Alemanha e Itália, onde $70 \%$ a $90 \%$ de sua população têm usado esses recursos da medicina tradicional sob as denominações: complementar, alternativa ou não convencional (OMS, 2011).

No SUS, as ações/programas com plantas medicinais e fitoterapia, distribuídos em todas as regiões do país, ocorrem de maneira diferenciada, em virtude dos diferentes biomas (Brasil, 2012). Muitos foram os avanços nas últimas décadas com a formulação e implementação de políticas públicas, programas e legislação com vistas à valoração e valorização das plantas medicinais.
Atualmente, os principais instrumentos norteadores para o desenvolvimento das ações/programas com plantas medicinais e fitoterapia são: Política Nacional de Práticas Integrativas e Complementares no SUS (PNPIC), com diretrizes e linhas de ação para "Plantas Medicinais e Fitoterapia no SUS", e Política Nacional de Plantas Medicinais e Fitoterápicos (PNPMF), com abrangência da cadeia produtiva de plantas medicinais e fitoterápicos. Essas políticas foram formuladas em consonância com as recomendações da OMS; os princípios e diretrizes do SUS; o potencial e oportunidades que o Brasil oferece para o desenvolvimento do setor; a demanda da população brasileira e necessidade de normatização das experiências existentes no SUS. (Brasil, 2012).

O objetivo do estudo é analisar a Política Nacional de Práticas Integrativas e Complementares (PNPIC) publicada através de Portaria do Ministério da Saúde e a Política Nacional de Plantas Medicinais e Fitoterapia (PNPMF), instituída através de Decreto Presidencial, como uma contribuição ao entendimento das conquistas alcançadas, mas, sobretudo, do que ainda é necessário ser esclarecido para formulação de políticas específicas do setor no Brasil.

\section{Metodologia}

Para realização deste trabalho, utilizou-se a abordagem exploratória descritiva. Utilizou-se como fonte um levantamento dos documentos que definem as Políticas Nacionais de Práticas Integrativas e Complementares (PNPIC) e Política Nacional de Plantas Medicinais e Fitoterápicos (PNPMF).

\section{Resultados e Discussão}

A Política Nacional de Práticas Integrativas e Complementares no SUS (PNPIC), aprovada via Portaria Ministerial no 971 de 3 de maio de 2006 
apresenta-se como um instrumento que reúne as diretrizes do SUS e uma proposta inovadora que, de um lado respeita práticas de saúde não convencionais e, por outro, traz uma concepção ampliada do processo saúde-doença, visto que reconhece $o$ importante papel do usuário do serviço como ator e participante desta dinâmica (Brasil, 2006a).

A PNPIC é uma política de caráter nacional, voltada principalmente para a Atenção básica em saúde e recomenda a implementação de ações e serviços no SUS que tenham o cuidado continuado, humanizado e integral. Seu principal objetivo é garantir a prevenção de agravos, a promoção e a recuperação da saúde. A Portaria preconiza o aumento da resolubilidade do sistema com qualidade, eficácia, eficiência, segurança, sustentabilidade e, ainda, controle e participação social. A PNPIC procura extrapolar o entendimento medicalizado da saúde, observando os sujeitos em suas dimensões física, social, cultural e psicológica. A PNPIC foi formulada, aprovada e implementada de forma transversal e com a participação efetiva da sociedade, a fim de trazer avanços para a saúde do País. E a partir da elaboração da Política Nacional de Plantas Medicinais e Fitoterápicos, com diretrizes e ações para toda a cadeia produtiva, fortalecer as ações/serviços com plantas medicinais e fitoterapia.

A Política Nacional de Plantas Medicinais e Fitoterápicos (PNPMF), aprovada por meio do Decreto №5.813, de 22 de junho de 2006, estabelece diretrizes e linhas prioritárias para o desenvolvimento de ações, voltadas à garantia de acesso seguro e uso racional de plantas medicinais e fitoterápicos no Brasil, ao desenvolvimento de tecnologias e inovações, assim como ao fortalecimento das cadeias e dos arranjos produtivos, ao uso sustentável da biodiversidade brasileira e ao desenvolvimento do Complexo Produtivo da Saúde (Brasil, 2006b).
Esta política foi elaborada para estabelecer as diretrizes para a atuação do governo na área de plantas medicinais e fitoterápicos e constitui parte essencial das políticas públicas de saúde, meio ambiente, desenvolvimento econômico e social como elementos fundamentais de transversalidade na implementação de ações capazes de promover melhorias na qualidade de vida da população brasileira (Brasil, 2006b). Alguns princípios nortearam sua elaboração, tais como melhoria da atenção à saúde, uso sustentável da biodiversidade brasileira e fortalecimento da agricultura familiar, geração de emprego e renda, desenvolvimento industrial e tecnológico e perspectiva de inclusão social e regional, além da participação popular e controle social. Entre os fatores previamente admitidos, ressalta a necessidade de minimizar a dependência tecnológica do país e de estabelecer uma posição de destaque do Brasil no cenário internacional.

Por outro lado, PNPMF e a PNPIC trazem em suas diretrizes a promoção e inclusão da agricultura familiar nas cadeias produtivas de plantas medicinais, insumos e fitoterápicos, pois a maioria da matéria prima utilizada na produção de medicamentos é importada. A falta de incentivos para o setor ainda não foi resolvida, ainda apresenta muitos desafios a enfrentar como o fortalecimento institucional a Estados e Municípios e, sobretudo, a definição de recursos específicos para o desenvolvimento das diretrizes dessas políticas.

\section{Conclusões}

As políticas públicas e os problemas inerentes à sua implementação têm se constituído, nos últimos anos, em um tema recorrente no Brasil, porém não têm merecido a necessária atenção de modo a tornar-se um tema da agenda política nacional. 
Conclui-se que a implementação da PNPMF, em âmbito nacional avançou mais que a PNPIC, no que diz respeito à elaboração das normas e à regulamentação, por garantir à população o acesso seguro, eficaz e de qualidade, além de promover o uso racional das plantas medicinais e fitoterápicos em quaisquer das formas ofertadas: planta medicinal in natura, droga seca, fitoterápico manipulado e/ou industrializado.

Ambas avançaram na ampliação da oferta de serviços e de produtos da fitoterapia na rede pública e impactaram na aprovação de políticas públicas do setor.

\section{Referências}

BRASIL, 2006a. Ministério da Saúde. Gabinete do Ministro. Portaria no 971, de 3 de maio de 2006. Aprova a Política Nacional de Práticas Integrativas e Complementares no SUS. Diário Oficial da União, Brasília, DF.
BRASIL, 2006b. Ministério da Saúde. Decreto $\mathrm{n}^{0}$ 5.813, de 22 de junho de 2006. Aprova Política Nacional de Plantas Medicinais e Fitoterápicos. Diário Oficial da União, Brasília, DF.

BRASIL, 2012. Ministério da Saúde. Práticas integrativas e complementares: plantas medicinais e fitoterapia na atenção básica. Brasília: Ministério da Saúde. (Série A. Normas e Manuais Técnicos) (Cadernos de Atenção Básica; n. 31).

OMS 2011. ORGANIZAÇÃO MUNDIAL DA SAÚDE. Medicines: International Cooperation and Harmonization. Organização Mundial da Saúde; 2011 [23 de outubro de 2014]. Disponível em: http://www.who.int/medicines/areas/quality_safety/re gulation_legislation/harmonization/en/index.html

RATES, S.M.K. 2001. Plants as source of drugs. Toxicon v.39 p.603-613.

WILSON, E.O. 1997. A situação atual da diversidade biológica. In: Biodiversidade. Rio de Janeiro: Nova Fronteira, p.3-24. 